\title{
A clinical pathway through substance use disorder, first episode psychosis to post traumatic stress disorder on the basis of HPA axis dysfunction
}

\begin{abstract}
First episode psychosis (FEP) is frequently seen in the context of substance use disorder. This is typically viewed as evidence of a concurrent disorder in clinical practice. This paper purports that addiction, partially through causing pathological neuroadaptation of the hypothalamic pituitary adrenal (HPA) axis and abnormal stress response, predisposes clients to experiencing a discrete psychotic episode in recovery. Treating this scenario as a concurrent disorder may not address the psychological and physiological trauma of the FEP itself. This may lead to longstanding post-traumatic stress and poorer recovery outcomes.
\end{abstract}

Keywords: addiction, post traumatic stress, cortisol, neuroendocrine, first episode psychosis, HPA axis
Volume 2 Issue 2 - 2015

\section{Christopher William Ashton, Denise Duffie}

Harbour Front Health Group, Canada

Correspondence: Christopher William Ashton, HarbourFront Health Group, 346 Chapel Street, Grand Falls, New Brunswick, E3Z 2M4, Canada,Tel 506475 8989, Email chrisashton@hfhg.ca

Received: October 30, 2014 | Published: May 25, 2015
Abbreviations: PTSD, post traumatic stress disorder; SUDs, substance abuse disorders; FEP, first episode psychosis; HPA, hypothalamic pituitary adrenal

\section{Introduction}

Increasing attention, both in research circles and therapeutic environments, is being given to the association between and potential efficacy of co-treatment for addiction and post-traumatic stress disorder (PTSD). ${ }^{1}$ Recent studies indicate a high prevalence of PTSD clients among clients receiving treatment for substance abuse disorders (SUDs) ${ }^{2,3}$ while not addressing physiological similarities. Additionally, strong associations between cannabis and other substance abuse and first episode psychosis (FEP) have been reported. ${ }^{4,5}$ Similarities in behaviors and reported symptomatology among people with SUD, PTSD and FEP either in combinations or for single entity diagnosis are clinically significant. As well, associations between addiction and other affective and schizoid disorders including bipolar affective disorder and schizophrenia are strong and well reported. ${ }^{6}$ This further complicates effective assessment, treatment and ongoing management of clients with SUD. In particular, conclusions easily drawn for SUD clients along any stage of the disorder who experience an FEP can lead to potential misdiagnosis as a concurrent disorder, rather than a potential complication of SUD. At this stage the client is confronted with all the ramifications, of two (versus one), long term chronic diseases. Symptomatology following FEP is remarkably similar to that reported both in bipolar affective disorder with depression and suicidal ideation/behavior, as well as in PTSD, such as "living in permanent fear of the next one (psychotic episode)" and attendant avoidance and social withdrawal. ${ }^{7}$ Among other commonalities, all three conditions include a relationship with stress and its biopsychological implications. Abnormal basal cortisol levels and reactivity are implicated in SUD, PTSD and FEP as a result of hypothalamic-pituitary-adrenal (HPA) axis dysfunction. ${ }^{8-10}$ This finding and an understanding of potential mechanisms implicated within each entity are explored to further illuminate associated risk factors, as well as a clinical pathway among and co-existence of these conditions. Finally, the possibility of an FEP resulting as a discrete experience from SUD, rather than an additional disorder, is discussed.

\section{Discussion}

\section{Substance use disorder}

To manage the complexities surrounding these three entities, this paper will speak only to substance dependence as that component of SUD and addiction discussed herein. According to the DSM IV TR, substance dependence is a maladaptive pattern of use that is characterized by three or more of the following:

i. tolerance of the substance

ii. Withdrawal symptoms when the substance is reduced or ceased

iii. Using more than was planned or for a longer period than was planned

iv. Unsuccessful efforts to reduce or control use

v. Significant time spent obtaining, using, or recovering from use

vi. Interference with important social, occupational, or recreational activities

vii. Continued use despite knowledge of its cause or exacerbation of a physical or psychological problem(s). ${ }^{1}$

This dependence on exogenous substances causing neuroadaptation is thought fundamental to the pathophysiology and definition of addiction. ${ }^{11-13}$ Specifically, this neuroadaptation refers to the "hijacking" of the normal reward system in the brain through amplifying the deeper subconscious brain regions' stimulus for reward seeking, while impeding the executive inhibitory function which would prevent further indulgences. ${ }^{12}$ It is not unusual for addiction clients to attribute their use of exogenous substances as a method to medicate stress. Not surprisingly, abnormalities in the neuroendocrine 
stress response system through the hypothalamic-pituitary-adrenal (HPA) axis are implicated in substance use disorders. ${ }^{14}$ The HPA axis is "extremely sensitive to inputs from the limbic system and prefrontal cortex, two brain areas that are important in modulating reinforcement and motivational processes" including addiction. Active SUD blunts the HPA axis' reactivity; this represents a secondary outcome potentially reinforcing addictive behavior if one adopts the self medication hypothesis with clients seeking lower stress levels.

As stated by McEwen ${ }^{15}$ "the brain is the key to interpreting and responding to potentially stressful events." This paper purports that substances impair both cognitive input as well as interpretation of stressful events; the major reason behind SUD according to the self medication hypothesis. Neuroadaptation can then be expected in SUD at least in those areas associated with the stress response which are believed to have plasticity, namely the HPA axis. Given substances diminishing the stress response, with chronic use, it would then be expected that the HPA axis reactivity becomes blunted in accordance with impaired inputs and interpretations. A rebound effect is surmised on abstinence: this axis activity may become hyper-reactive (increasing cortisol secretion upon stress stimulus) as is experienced in most withdrawal syndromes, prior to returning to stable activity levels at some later, unspecified time. ${ }^{16}$ It is noteworthy that increased HPA axis activity is associated with increased dropout rates from residential care settings and, hence, poorer recovery outcomes. ${ }^{16,17}$ Given the contribution of increased HPA axis reactivity, in particular when coupled with low awakening cortisol levels, to negative feelings including pain, anxiety and depressed mood, the inability to withstand these negative feelings may explain the increase in treatment attrition..$^{14,18,19}$

\section{Post-traumatic stress disorder}

Associated with HPA axis dysfunction is also post-traumatic stress disorder (PTSD). Following exposure to a traumatic event, PTSD manifests as a chronic anxiety disorder, characterized by three sets of symptomatology: involuntary "re-experiencing the traumatic event (e.g. intrusive thoughts, nightmares), cognitive or behavioral avoidance of reminders of the event, and physiological hyperarousal". ${ }^{10}$ In general, PTSD has been found to be associated with dysfunctional HPA axis function with decreased basal cortisol levels as well as exaggerated cortisol secretion in stressful situations. As such, dysfunctional HPA axis activity is similar in both PTSD and addiction, particularly in early abstinence whereby an excessive cortisol response to stress is found to be an obstacle to recovery. Emerging evidence shows a strong link between PTSD and addiction. Gielen et al. ${ }^{2}$ in a study of 423 patients with SUD and 206 healthy control subjects found an incidence of PTSD of $36.6 \%$ in the SUD group compared to $10.6 \%$ for the controls. ${ }^{2}$ Exposure to prior trauma was found to be $97.4 \%$ and $86.3 \%$ for the SUD and control groups respectively. While the incidences for PTSD and trauma were somewhat elevated in this study for the control group, the incidence of PTSD for SUD subjects was found to be in keeping with previous studies which demonstrated rates of 25.3 to $41 \%$. This study also found that PTSD was best diagnosed through standardized measurement tools, rather than by clinical impressions lending to the implication that PTSD may be under-diagnosed by clinicians attending to SUD clients.

Van Dam et al. ${ }^{3}$ speak to the evidence and theory surrounding posttraumatic stress and substance abuse disorders which indicates a higher relapse rate when the disorders are concurrent. A higher incidence of relapse following traditional, non-traumatic focus treatment for SUD, while helping both SUD and PTSD symptoms, has been demonstrated to result primarily from the re-emergence of PTSD symptoms following initial addiction treatment. It follows logically that without the psychoactive substances medicating the painful symptomatology, cognitive and behavioral aspects of PTSD may re-emerge. Research exists which shows that "trauma-related cues can trigger a craving response". ${ }^{3}$ From the standpoint of aftercare, in addition to contributing to psychic pain, chronic depression and anxiety, PTSD often confines the affected client to a very small, isolated existence due to the underlying psychopathology. This motivates the sufferer to avoid all potential exposures to experiences which could be associated with the traumatic event. This tendency towards social isolation may present a considerable psychological barrier to participating in recovery activities, particularly with groups of people in aftercare aimed at achieving life satisfaction without substances. Van Dam et al. ${ }^{3}$ also acknowledge that people with substance abuse disorder have a higher risk of trauma exposure due to behavioral risks surrounding and resulting from addiction. As such, the authors maintain that SUD may precede PTSD although the inverse temporal relationship is usually the norm. Traumatic exposure is a common platform found among PTSD and addiction clients; nearly $100 \%$ of those suffering from either condition report exposure(s) to significant traumatic events. With that being said, a multitude of questions remain as to why PTSD and addiction occurs in a relative minority of people in society with trauma exposures being so highly prevalent among population members of North America. A common link appears to be similar dysfunctions of the HPA axis among those with PTSD, SUD or both. As exemplified in withdrawal and early recovery for SUD, both conditions exhibit hyperactive cortisol secretion with stress. Unto itself, HPA axis dysfunction and hyper reactivity under stress are associated with, and believed contributory to, a wide spectrum of pain, anxiety and depression syndromes similar to those reported by SUD and PTSD clients. ${ }^{19}$ If one assumes some veracity of the self medication hypothesis of addiction, ample discomfort exists within those with HPA axis dysfunctional one to potentially use substances to manage their dysphoria.

\section{First episode psychosis}

While acknowledging that the discussion of PTSD and SUD is adequately complex unto itself, the issue of first episode psychosis (FEP) occurring in recovery will be discussed in relation to the other two entities. It is well accepted that high rates of substance abuse are coincident with other psychiatric conditions. Over half of clients with bipolar I disorder have a history of substance abuse. ${ }^{6}$ The substances most commonly abused substances for those diagnosed with BPI are, in decreasing prevalence, alcohol, cocaine, opiates, polysubstances and sedative/hypnotics. Conversely, a significant proportion of those presenting with current or a past history of SUD have an associated diagnosis of bipolar disorder. Nallet et al. ${ }^{6}$ present evidence reporting that "a lifetime history of mania was reported in $6.5 \%$ of alcoholic men and $10.6 \%$ of alcoholic women." According to DSM IV TR, the diagnosis of bipolar I disorder rests on a history of mania. Specifically, the "essential feature of Bipolar I Disorder is a clinical course that is characterized by the occurrence of one or more Manic Episodes or Mixed Episodes". ${ }^{20}$ In turn, criteria for a manic episode are:

A. "A distinct period of abnormally and persistently elevated, expansive, or irritable mood, lasting at least 1 week (or any duration if hospitalization is necessary) 
B. During the period of mood disturbance, three (or more) of the following symptoms have persisted (four if the mood is only irritable) and have been present to a significant degree:

i. Inflated self-esteem or grandiosity

ii. Decreased need for sleep (e.g., feels rested after only 3 hours of sleep)

iii. More talkative than usual or pressure to keep talking

iv. Flight of ideas or subjective experience that thoughts are racing

v. Distractibility (i.e., attention too easily drawn to unimportant or irrelevant external stimuli)

vi. Increase in goal-directed activity (either socially, at work or school, or sexually) or psychomotor agitation

vii. Excessive involvement in pleasurable activities that have a high potential for painful consequences (e.g., engaging in unrestrained buying sprees, sexual indiscretions, or foolish business investments).

C. The symptoms do not meet criteria for a Mixed Episode.

D. The mood disturbance is sufficiently severe to cause marked impairment in occupational functioning or in usual social activities or relationships with others, or to necessitate hospitalization to prevent harm to self or others, or there are psychotic features.

E. The symptoms are not due to the direct physiological effects of a substance (e.g., a drug of abuse, a medication, or other treatments) or a general medical condition (e.g., hyperthyroidism).

Note: Manic-like episodes that are clearly caused by somatic antidepressant treatment (e.g., medication, electroconvulsive therapy, light therapy) should not count toward a diagnosis of Bipolar I Disorder. ${ }^{20}$

As such, while bipolar I disorder and SUD are strongly associated, criteria for a manic episode are exclusive of physiological effects of substances. Nonetheless, episodes of mania encountered in withdrawal from substances, particularly opiates, are frequently encountered and a subsequent diagnosis of bipolar disorder has been attributed to these patients. ${ }^{21}$ To complicate matters further, the lines of differentiation among bipolar I disorder, mania, psychosis and schizophrenia are blurred. ${ }^{22}$ Specifically, reporting of visual or auditory hallucinations as criteria for diagnosis of psychosis in manic episodes may be difficult to accurately differentiate, particularly given the client's strong reluctance to discuss psychotic episodes and resulting confusion in attempting to do so. ${ }^{7}$ Standard clinical preference is to err on the side of safety in caring for this group of patients who collectively present with a significant risk of suicide as well as a tremendous desire to avoid a repeat psychotic episode. ${ }^{23}$ As such, it is understandable that a manic or psychotic episode in the context of substance abuse disorder would result in a diagnosis of bipolar or schizophrenia and the administration of anti-psychotic medications. Unfortunately, this clinical scenario has now become one of concurrent disorders, rather than a potential event related to SUD and recovery; concurrent disorders are associated with much poorer outcomes for those seeking to recover from addiction. Revisiting the DSM IV TR criteria for bipolar I disorder and the manic event, caution is given to exclude manic symptoms "due to the direct physiological effects of a substance (e.g., a drug of abuse, a medication or other treatment) or a general medical condition (e.g., hyperthyroidism) ". ${ }^{20}$ Much of the literature in this regard speaks to the association between acute withdrawal syndrome from substances and psychotic states or associations of psychosis with substances, cannabis in particular. ${ }^{5,21}$ The emergence of a first episode of psychosis occurring well beyond the acute withdrawal phase may still represent a remaining phenomenon of the physiologic effects of substances. It is particularly illuminating to examine this through the lens of neuroadaptation and the stress vulnerability model. ${ }^{22,23}$ Accepting that SUD has caused neurodaptation of the HPA axis and stress response, the abstinent client is now facing 'life on life's terms' in the frequently stressful environment of recovery. Maladaptation of the HPA axis could be argued to increase clients' vulnerability to highly abnormal stress responses: the threshold for first onset of psychosis is accordingly lowered. ${ }^{25}$ Hence, the clinical tendency to diagnose FEP in the context of SUD as evidence of a concurrent disorder merits reconsideration.

Recent research ${ }^{7,24,25}$ has illuminated the subjective sequel experienced by clients following an FEP. They can be devastating; in one study, $77 \%$ thought they had suffered a persistent loss, change or disruption to their life as a result of their psychotic episode. Additionally, $60 \%$ of subjects reported thinking that "their hopes and aspirations were no longer achievable". ${ }^{24} 40 \%$ of those affected reported suicidal ideation with $31 \%$ of those attempting. Study participants reported "living in permanent fear of the next" psychotic episode following their first experience. ${ }^{7}$ Implications of this experience of permanent fear can be profound, affecting clinical impressions and management including pharmacologic and self care. The belief in the definitive probability of experiencing another psychotic episode on the part of clients is aligned with clinical impressions that FEP in the context of SUD, is diagnostic for a chronic relapsing psychiatric disease. Patients could expectedly be reluctant to question a diagnosis of a concurrent disorder and to accept the value of antipsychotic medication. Exploring the possibility of FEP as a discrete event following treatment for SUD, rather than a manifestation of bipolar I or schizoaffective disorder, necessitates consideration of the effects of SUD as outlined in the DSM. Post SUD withdrawal, HPA axis activity is associated with hyperactive secretion and, hence, abnormal physiologic stress responses. ${ }^{26}$ Recovery itself from addiction represents a highly stressful experience for many and one fraught with high levels of relapse. The functioning of the body's neuroendocrine stress response in FEP is reported as dysfunctional and, similar to SUD, exhibits HPA axis hyper reactivity. ${ }^{27}$ Additionally, FEP is thought to emerge, given the requisite diathesis, following a period of high stress as could be encountered in the recovery experience. Dysfunctional physiological stress responses resulting from SUD could well represent a significant component of the precursor to the FEP experience. The important commonality seen in clients following a FEP event, living in permanent fear of another episode, lends itself to anxiety and behaviors such as social isolation frequently encountered in PTSD. Indeed, Phassouliotis et al. ${ }^{26}$ report on the high incidence of PTSD following FEP, as well as the similar neuroendocrine abnormalities. ${ }^{27}$

\section{Conclusion}

Abnormal hypothalamic-pituitary-adrenal axis function may well represent a common platform to explain the relationships and 
potential cascade of morbidity arising through substance abuse disorder and recovery precipitating an FEP; untreated, the psychotic episode experience may well progress into a form of post-traumatic stress disorder. While PTSD is generally associated with external events, discussions of PTSD arising from traumatic internalizing experiences including FEP have been reported. ${ }^{24}$ Implications of incorrectly diagnosing a dual disorder following a FEP in SUD recovery are significant. While acknowledging the relative safety of treating FEP following attainment of abstinence for SUD as representing a concurrent disorder, the ramifications of diagnosis are enormous compared to the possibility of considering FEP as a discrete (non-repeating) event in recovery. In addition to potentially needless exposure to cognition reducing, anti-psychotic medications and disability associated with concurrent disorder, usual care does not generally explore or treat the devastating effects of the FEP itself. ${ }^{24} \mathrm{As}$ such, these clients are more likely to acquire PTSD for the long term, unless specifically or otherwise addressed through additional recovery supports. Prognosis regarding recovery itself would be expectedly reduced.

The literature regarding the potential cascade of adverse events, from SUD to FEP followed by PTSD, for those in recovery from SUD is sparse, if not absent. Implications of this hypothesis in addictions could be highly significant in assessment, early treatment and aftercare. The argument presented herein suggests that mental health professionals and researchers reconsider standard practice for those who experience an FEP over the course of recovery from SUD. Unfortunately, there is an absence of high quality, long term randomized controlled trials aimed at exploring the validity of this hypothesis, as well as potential significant risks involved in not treating as a dual disorder. While close monitoring and care, particularly following the FEP, and ongoing assessment to pharmacologically intervene, only in the case of a further prodrome or psychotic episode, holds significant promise. Much further study is recommended, prior to embarking on this course of care.

\section{Acknowledgements}

None.

\section{Conflict of interest}

Author declares that there is no conflict of interest.

\section{References}

1. McCauley J, Killeen T, Gros D, et al. Posttraumatic stress disorders and co-occurring substance abuse disorders: advances in assessment and treatment. Clin Psychol. 2012;19(3).

2. Gielen N, Havermans R, Tekelenburg M, et al. Prevalence of posttraumatic stress disorder among patients with substance use disorder: it is higher than clinicians think it is. Eur J Psychotraumatol. 2012;3.

3. Van Dam D, Ehring T, Vedel E, et al. Trauma-focused treatment for posttraumatic stress disorder combined with CBT for severe substance use disorder: a randomized controlled trial. BMC Psychiatry. 2013;13:172.

4. De Ruiter W, Cheng C, Gehrs M, et al. Substance abuse and smoking among a Canadian cohort of first episode psychosis patients. Community Ment Health J. 2013;49(6):815-821.

5. Dragt S, Nieman D, Schultze-Lutter F, et al. Cannabis use and age at onset of symptoms in subjects at clinical high risk for psychosis. Acta Psychiatr Scand. 2012;125(1):45-53.
6. Nallet A, Weber B, Favre S, et al. Screening for bipolar disorder among outpatients with substance use disorders. Eur Psychiatry. 2013;28(3):147-153.

7. Sandhu A, Ives J, Birchwood M, et al. The subjective experience and phenomenology of depression following first episode psychosis: A qualitative study using photo-elicitation. $J$ Affect Disord. 2013;149(1-3):166-174.

8. Borges S, Gayer-Anderson C, Mondelli V. A systematic review of the activity of the hypothalamic-pituitary-adrenal axis in first episode psychosis. Psychoneuroendocrinology. 2013;38(5):603-611.

9. Boschlooa L, Vogelzangsa N, Lichta M, et al. Heavy alcohol use, rather than alcohol dependence, is associated with dysregulation of the hypothalamic-pituitary-adrenal axis and the autonomic nervous system. Drug Alcohol Depend. 2011;116(1-3):170-176.

10. Edmondson D, Cohen B. Posttraumatic stress disorder and cardiovascular disease. Prog Cardiovascular Diseases. 2013;55(6):548-556.

11. Kalant $\mathrm{H}$. What neurobiology cannot tell us about addiction. Addiction. 2009;105(5):780-789.

12. Karim R, Chaudri P. Behavioral addictions: an overview. J Psychoactive Drugs. 2012;44(1):5-17.

13. Sellman D. The 10 most important things known about addiction. Addiction. 2010;105(1):6-13.

14. Lorenzetti V, Lubman D, Velakoulis D, et al. Pituitary gland volume among heroin users stabilised on substitution pharmacotherapy. Drug Alcohol Depend. 2010;110(1-2):164-166.

15. McEwen B. The neurobiology of stress: from serendipity to clinical relevance. Brain Res. 2000;886(1-2):172-189.

16. Arbour S, Hambley J, Ho V. Predictors and Outcome of Aftercare Participation of Alcohol and Drug Users Completing Residential Treatment. Subst Use Misuse. 2011;46(10):1275-1287.

17. Daughters S, Richards M, Gorka SM, et al. HPA axis response to psychological stress and treatment retention in residential substance abuse treatment: A prospective study. Drug Alcohol Depend. 2009;105(3):202-208.

18. McBeth J, Silman J, Gupta A, et al. Moderation of psychosocial risk factors through dysfunction of the hypothalamic-pituitary-adrenal stress axis in the onset of chronic widespread musculoskeletal pain: findings of a population-based prospective cohort study. Arthritis Rheum. 2007;56(1):360-371

19. Thornton LM, Andersen BL, Blakely WP. The pain, depression, and fatigue symptom cluster in advanced breast cancer: covariation with the hypothalamic-pituitary-adrenal axis and the sympathetic nervous system. Health Psychol. 2010;29(3):333-337.

20. American Psychiatric Association. Diagnostic and statistical manual of mental disorders. 4th ed. text rev, Washington, DC, USA; 2000.

21. Shariat SV, Hosseinifard Z, Taban M, et al. Mania Precipitated by Opioid Withdrawal: A Retrospective Study. Am J Addict. 2013;22(4):338-343.

22. Gispen-de Wied C, Jansen L. The Stress-vulnerability Hypothesis in Psychotic Disoders: Focus on the Stress Response Systems. Curr Psychiatry Rep. 2002;4(3):166-170.

23. Goh C, Agius M. The Stress Vulnerability Model: How does Stress Impact on Mental Illness at the Level of the Brain and What are its Consequences? Psychiatr Danub. 2010;22(2):198-202.

24. Domján N, Csifcsák G, Drótos G, et al. Different patterns of auditory information processing deficits in chronic schizophrenia and bipolar disorder with psychotic features. Schizophr Res. 2012;139(1-3):253-259. 
25. Tarrier N, Khan S, Cater J, et al. The subjective consequences of suffering a first episode psychosis: trauma and suicide behavior. Soc Psychiatry Psychiatr Epidemiol. 2007;42(1):29-35.

26. Phassouliotis C, Garner AB, Phillips LJ, et al. Enhanced cortisol suppression following administration of low-dose dexamethasone in firstepisode psychosis patients. Aust N Z J Psychiatry. 2013;47(4):363-370.
27. MacBeth A, Gumley A, Schwannauer M, et al. Service engagement in first episode psychosis. Clinical and premorbid correlates. J Nerv Ment Dis. 2013;201(5):359-364 